\title{
Treatment of hepatic metastases by cryotherapy and regional cytotoxic perfusion
}

Cryotherapy was probably first used to treat tumours by Arnott ${ }^{1}$ almost 150 years ago. Much scientific work was done more than 20 years ago, including findings on the effect of repeated freeze/thaw cycles in the liver. ${ }^{2}$ Hepatic cryotherapy was done by direct application of liquid nitrogen at $-196^{\circ} \mathrm{C}$, or by surface coils with good results. ${ }^{3}$ While these techniques were applicable to hepatocellular carcinoma, multiple metastases present a different problem. We have used a triple lumen insulated probe system (Cryotech) of 5 or $9 \mathrm{~mm}$ diameter, designed to allow the destruction of multiple lesions within the liver without much overlying liver tissue. ${ }^{+}$ Intraoperative contact ultrasound is an essential part of the technique for identifying and localising tumours, aiding probe placement, as well as controlling the extent of the freezing process. ${ }^{5}$

Thirty eight of our 46 patients in Australia to date have had metastatic colon cancer, as was the case in our Nottingham series, ${ }^{6}$ although hepatocellular cancer, neuroendocrine tumours, ovarian cancer, and malignant melanoma have also been treated.

The selection of patients is undoubtedly important. We have regarded extrahepatic disease as a contraindication (except, perhaps, for porta hepatis nodes within the perfusion territory of our catheter system) and actively look for pulmonary, bone, and retroperitoneal lymphatic disease before operation. The number and size of lesions that can be treated is currently limited by time and equipment design but a $50 \mathrm{~mm}$ diameter iceball can be created in a perfused liver in approximately 15 minutes; larger lesions require multiple probes. All areas of the liver are easily accessible to treat.

While cryotherapy may, in the future, become an alternative to liver resection, in fit patients we recommend liver resection for suitable lesions. In patients with a limited number - perhaps four or less lesions that are unresectable because of position or distribution - then destruction by cryotherapy may offer results just as good as if resection were possible.

It is undoubtedly in patients with more than four lesions that treatment is most controversial: there is, however, now no doubt that serum concentrations of carcinoembryonic antigen (CEA) can be profoundly reduced by cryotherapy in such patients, ${ }^{6}$ but does this matter? Serum CEA (in tumours that secrete) does correlate well with liver tumour bulk and, in patients with untreated hepatic metastases, does increase inexorably. Reduction of CEA after chemotherapy is associated with significantly increased survival, ${ }^{7}$ but it remains to be seen whether the reduction in CEA after cryotherapy will be translated into improved survival.

The use of 'adjuvant' hepatic chemotherapy after cryotherapy seemed logical, although it had not previously been reported. There are several good examples of other tumours where results of chemotherapy correlate with the volume of residual disease, and there is a strong suggestion that results of hepatic artery chemotherapy for metastatic colon cancer also depend on percentage hepatic replacement, ${ }^{8}$ so destroying all tumour that can be detected intraoperatively (hepatic cytoreduction) followed by 'adjuvant' infusion hepatic artery chemotherapy could offer advantage. When restarting this programme in Australia, we decided to add infusion hepatic artery chemotherapy to try to achieve longer suppression of CEA. Our regimen includes $50 \mathrm{mg}$ folinic acid by intraarterial bolus, followed by 5-FU ( $1 \mathrm{~g})$ over 24 hours for four days each month.

Safety of hepatic cryotherapy is good: none of our patients have died during operation or in the postoperative period, although there have been, we believe, at least three deaths in other centres due to coagulopathy, sepsis (colonic injury), and liver failure (hepatic vein thrombosis). Morbidity has also been limited. About one third of our patients required blood transfusion. Perhaps the most worrying problem has been the frequent myoglobinuria and clinical renal failure reported by the Pittsburgh group. ${ }^{5}$ We have only seen transient renal impairment in two patients, one of whom had a major intraoperative anaphylactic episode; the other had pre-existing renal impairment. Hospital stay has been short, at around six days: respiratory complications, with occasionally significant right sided pleural effusion, were the commonest complications we have seen. A significant fall in platelet count is regularly seen' but only one patient has had postoperative bleeding because of disseminated intravascular coagulation. Liver sepsis has not been seen in the postoperative period. The healing of the cryolesion has been monitored radiographically: initially a small amount of central gas may be seen with a large surrounding avascular area corresponding to the position of the iceball. This undoubtedly shrinks but a 'lesion' remains, which can be difficult to differentiate from tumour. In animals ${ }^{10}$ and in patients on whom we have reoperated after cryotherapy, a spherical area of yellowish green fibrous tissue is seen.

The quality of life of our patients has been good, unless and until they have developed further metastatic disease. Failure, in our experience, has most commonly been extrahepatic - in the lungs and bone - although one patient, in whom the gall bladder was not removed because of local infiltration, developed severe cholecystitis with treatment, which prevented further treatment and he died rapidly of progressive liver disease. Of the 13 deaths in the 46 Australian patients to date, 10 were from systemic - mainly bone and lung disease, although local failure certainly still occurs. The high incidence of bone and lung disease we have seen may reflect a change in the natural history of the disease in patients who may previously have died from their multiple hepatic metastases. Fortunately, not all patients have such systemic disease. The role of adjuvant systemic treatment for the possible minimal systemic disease after cyrotherapy is clearly interesting.

In our total experience of over 67 procedures, 34 received infusion hepatic artery chemotherapy and experienced no excess morbidity, other than catheter related problems (thrombosis, displacement). CEA suppression seems to have occurred for considerably longer, in that, while $62 \%$ and $75 \%$ of colorectal cancer patients in the United Kingdom treated by cryotherapy alone had a relapse of their CEA to pretreatment values by three and six months respectively, in 34 patients who received adjuvant infusion hepatic artery chemotherapy after cryotherapy, only $14 \%$ and $15 \%$ have 
relapsed at the same time intervals to date. This is probably analogous to the findings of Kemeny's group, where infusion hepatic artery chemotherapy following liver resection achieve a longer tumour free period. ${ }^{1}$ An example of the beneficial effect of infusion hepatic artery chemotherapy after hepatic cryotherapy was recently described by Horton, ${ }^{12}$ where a catheter perfusing only the right lobe controlled further growth adequately, while disease progression occurred in the left, despite an initially satisfactory reduction in CEA.

Significant problems with the technique remain: cracking of the iceball is a not infrequent, tedious and haemorrhagic intraoperative complication, but can be easily managed by packing and suture. Techniques and equipment are still evolving. We recently compared single and double freeze/ thaw cycles. The latter achieved greater destruction, as measured by serum liver cell enzymes. The procedure is very slow, frequently taking more than four hours. ${ }^{13}$ The optimal method of control - ultrasound or needle thermocouples in the edge of the lesion - has not yet been established. While ultrasound accurately identifies the edge of the iceball, this does not correlate exactly with the eventual area of necrosis and the adoption of a $1 \mathrm{~cm}$ ultrasound margin ignores the great differences in temperature gradient across iceballs of varying diameter.

There are currently three other clinical techniques that can loosely be described as imaging controlled hepatic destruction: laser, ${ }^{14}$ alcohol injection, ${ }^{15}$ and interstitial radiotherapy. ${ }^{16}$ The first two, and potentially the third technique, can be done percutaneously, whereas cryotherapy at present requires an open operation. Hepatic cryotherapy, however, has now been used in several hundred patients, with great accuracy, safety, and some evidence of efficacy..$^{3513}$ Cryotherapy is much more easily controlled than thermal or injection techniques because the iceball is so easily seen on intraoperative ultrasound. There is also evidence from the sizeable experience of alcohol injection in hepatocellular carcinoma that this technique is best reserved for small $(2 \mathrm{~cm})$ diameter lesions. ${ }^{18}$

While we now know more about the safety and effects of hepatic cryotherapy on tumours - that it can profoundly reduce serum tumour markers and, when used with infusion hepatic artery chemotherapy, can achieve longterm suppression of disease - we do not yet know whether it can improve survival in patients with many metastases, or rival the results of surgery in patients with better prognostic lesions.

D L MORRIS

$M$ D A'C HORTON

A V DILLEY

A WARLTERS
P R CLINGAN

Department of Surgery,

University of New South Wales

and Oncology Department,
The St George Hospital,

Belgrave Street,

Kogarah, Sydney,

NSW 2217,

Australia

1 Arnott J. On the treatment of cancer by the regulated application of an anaesthetic

temperature. London: J Churchill, 1851:32-54.
Gill W, Fraser J, Carter DR. Repeated freeze/thaw cycles in cryosurgery. Nature 1968; 219: 410-3.

3 Zhou XD, Tang ZY, Yu YQ, Ma ZC. Clinical evaluation of cryosurgery in the treatment of primary liver cancer: report of 60 cases. Cancer 1988; 61 : $1889-92$.

4 Charnley RM, Morris DL. Cryotherapy for liver metastases: a new approach. Brf Surg 1989; 76: 1040-1.

5 Onik G, Rubinsky B, Zemel R, et al. Ultrasound guided hepatic cryosurgery in the treatment of metastatic colon carcinoma. Cancer 1991; 67: 901-7.

6 Charnley RM, Thomas M, Morris DL. Effect of hepatic cryotherapy on serum CEA concentration in patients with inoperable hepatic metastases from CEA concentration in patients with inoperable
colorectal cancer. Aust NZ f Surg 1990; 61: 55-8.

7 Quentmeier A, Schlag P, Hohenberger P, Schwartz V, Abel U. Assessment of serial carcinoembryonic antigen: determinations to monitor the therapeutic progress of metastatic liver disease treated by regional chemotherapy. f Surg Oncol 1989; 40: 112-8.

8 Hunt TM, Flowerdew ADS, Birch SJ, Williams JD, Mullee MA, Taylor I. Prospective randomised controlled trial of hepatic arterial embolisation or infusion chemotherapy with 5-fluorouracil and degradable starch microspheres for colorectal liver metastases. Br f Surg 1990; 77: 779-82.

9 Goodie DB, Horton MDA, Morris R, Nagy LS, Morris DL. The anaesthetic experience with hepatic cryotherapy for metastatic colonic carcinoma. Anaesth Intensive Care 1992; 20: 491-6.

10 Dilley AV, Warlters A, Gillies A, Dy DY, Morris DL. Hepatic cryotherapy: is portal clamping worth it? Aust NZ F Surg 1991; 61: A522.

11 Wagman LD, Kemeny M, Leong L, et al. A prospective randomised evaluation of the treatment of colorectal cancer metastatic to the liver. $\mathcal{F}$ Clin Oncol 1990

12 Horton MDAC, Warlters A, Dilley A, Clingan PR, Morris DL. Hepatic artery cytotoxic perfusion therapy after cryotherapy: a single patient control trial. Med F A ust 1991; 155: 849 .

13 Horton MDA, Warlters A, Dilley AV, Clingan PR, Morris DL. Survival after hepatic cryotherapy for hepatic metastases. Br F Surg 1992; 79: A452.

14 Masters A, Steger AC, Bown SG. Role of interstitial therapy in the treatment of liver cancer. Br f Surg 1991; 78: 518-23.

15 Livraghi T, Salmi A, Bolondi L, et al. Small hepatocellular carcinoma: percutaneous alcohol - results in 23 patients. Radiology 1988; 168: 313-7.

16 Nauta RJ, Heres EK, Thomas DS, et al. Intra-operative single dose radiotherapy. Arch Surg 1987; 122: 1392-5.

17 Ravikumar TS, Steele GD. Hepatic cryosurgery. Surg Clin North Am 1989; 69: 433-9.

18 Livraghi T, Festi D, Monti F, Salmi A, Vettori C. US guided percutaneous alcohol injection of small hepatic and abdominal tumors. Radiology 1986: 161: 309-12. 